\title{
SHUTTER-LESS TEMPERATURE-DEPENDENT CORRECTION FOR UNCOOLED THERMAL CAMERA UNDER FAST CHANGING FPA TEMPERATURE
}

\author{
Dong Lin ${ }^{\mathrm{a}, *}$, Patrick Westfeld ${ }^{\mathrm{a}}$, Hans-Gerd Maas ${ }^{\mathrm{a}}$ \\ ${ }^{a}$ Technische Universität Dresden, Institute of Photogrammetry and Remote Sensing, 01062 Dresden, Germany - \\ (dong.lin, patrick.westfeld, hans-gerd.maas)@tu-dresden.de
}

KEY WORDS: Uncooled Thermal Camera, Fast Changing FPA Temperature, Shutter-less, Temperature-dependent Correction, Radiometric Calibration, FLIR GEV

\begin{abstract}
:
Conventional temperature-dependent correction methods for uncooled cameras are not so valid for images under the condition of fast changing FPA temperature as usual, therefore, a shutter-less temperature-dependent correction method is proposed here to compensate for these errors and stabilize the camera's response only related to the object surface temperature. Firstly, sequential images are divided into the following three categories according to the changing speed of FPA temperature: stable $\left(0^{\circ} \mathrm{C} / \mathrm{min}\right)$, relatively stable $\left(<0.5^{\circ} \mathrm{C} / \mathrm{min}\right)$, unstable $\left(>0.5^{\circ} \mathrm{C} / \mathrm{min}\right)$. Then all of the images are projected into the same level using a second order polynomial relation between FPA temperatures and gray values from stable images. Next, a third order polynomial relation between temporal differences of FPA temperatures and the above corrected images is implemented to eliminate the deviation caused by fast changing FPA temperature. Finally, radiometric calibration is applied to convert image gray values into object temperature values. Experiment results show that our method is more effective for fast changing FPA temperature data than FLIR GEV.
\end{abstract}

\section{INTRODUCTION}

Thanks to the fast development of microbolometer focal plane array (FPA), thermography using uncooled thermal cameras has achieved more and more attention in recent years (Niklaus et al. 2007). Without the requirement for stabilized temperature sensor, higher spatial resolution, smaller detector pitch as well as lower power consumption could be designed. This makes thermal imagery more cost-effective, lighter and smaller (Bhan et al. 2009). Therefore, uncooled cameras are widely used in forest fire protection (Ambrosia et al. 2003), building thermal leakage detection (Hoegner et al. 2016, Westfeld et al. 2015), night vision (Liu et al. 2012), face recognition (Socolinsky et al. 2003), $\mathrm{CO}_{2}$ gas leakage from vegetation imagery (Johnson et al. 2012), water contamination monitoring (Lega et al. 2010).

In order to make any of the above applications viable, radiometric calibration which refers to forming a quantitative relation between image gray values and temperature or radiance of object has to be done accurately. However, the biggest drawback for uncooled thermal camera is that the camera output depends not only on the object radiance but also on the time-variant sensor temperature, which means the

\footnotetext{
* Corresponding author
} 
calibration parameters need to be updated almost continuously. Therefore, removing the image response from FPA temperature is important to measure the object temperature accurately.

One FPA temperature-dependent correction method is using one or more blackbodies to perform an online recalibration during measurement (Kruse et al. 2001). Although this approach holds for most of the satellite applications for example cloud imagery system (Thurairajah et al. 2005), it is not resource-efficient and practicable for terrestrial applications because there is no room for a large, heavy and expensive blackbody. In place of blackbody, shutter is widely used as an equivalent uniform temperature source to complete the radiometric calibration (Nugent et al. 2014). However, shutter-based compensation approaches have to close the shutter regularly during the measurement which leads to interruption and decrease of maximum acquisition rate, so they are not fit for seamless and real-time applications.

An alternative method is to combine FPA temperature with the updating of correction parameters (Budzier et al. 2015). There are several temperature-dependent correction methods available, such as Kalman filter (Torres et al. 2003), piecewise Lagrange interpolation (Liang et al. 2017) and multivariate regression model using multiple temperature inside the camera (Tempelhahn et al. 2016). The main drawback is that none of them take fast changing FPA temperature into consideration in their experiments. Fast changing FPA temperature is primarily influenced by ambient temperature as well as self-heating when used outdoors with wind or abrupt weather changes. Then, the original relation (known from laboratory calibration) between the FPA temperatures and the image gray values is not valid anymore, which would lead to wrong measurement without proper correction.

This paper proposes a novel shutter-less FPA temperature-dependent real-time correction method which is effective for fast changing FPA temperature data. The main idea of the method presented here is, firstly, all of the temperature-dependent models including stable temperature-dependent model, fast changing temperature-dependent model as well as radiometric calibration model are established by datadriven estimation for only one time off-the-line. Subsequently, on-the-line, all the acquired images are directly applied in object temperature retrieval by using the stored calibrated parameters as well as the temperature-dependent models.

\section{PRINCIPLE OF PROPOSED METHOD}

\subsection{Temperature-dependent Model Determination}

During the experiment, our thermal camera (FLIR Ax65, whose parameters are shown in Table 1) is put inside a chamber with ambient temperature changing from $10^{\circ} \mathrm{C}$ to $35^{\circ} \mathrm{C}$ and then back, viewing a four element Peltier blackbody (shown in Figure 1) which are set to $11.6^{\circ} \mathrm{C}, 26.4^{\circ} \mathrm{C}, 67.6^{\circ} \mathrm{C}$ and $36.8^{\circ} \mathrm{C}$ constantly. The maximum changing speed of FPA temperature is more than $1{ }^{\circ} \mathrm{C} / \mathrm{min}$, which is much more than the condition of other papers $\left(0.5^{\circ} \mathrm{C} / \mathrm{min}\right.$ in Nugent et al. 2013 and Nugent et al. 2014), but will probably happen when used outdoors.

\begin{tabular}{|c|c|}
\hline Spatial resolution/IFOV & $640 \times 512 / 45^{\circ} \times 37^{\circ}$ \\
\hline Lens & $13 \mathrm{~mm}$ \\
\hline Image frequency & $30 \mathrm{~Hz}$ \\
\hline Detector pitch & $17 \mu \mathrm{m}$ \\
\hline $\begin{array}{c}\text { Object temperature } \\
\text { range }\end{array}$ & $-25^{\circ} \mathrm{C}$ to $+135^{\circ} \mathrm{C}$ \\
\hline Weight & $200 \mathrm{~g}$ \\
\hline
\end{tabular}

Table 1. Parameters of FLIR Ax65

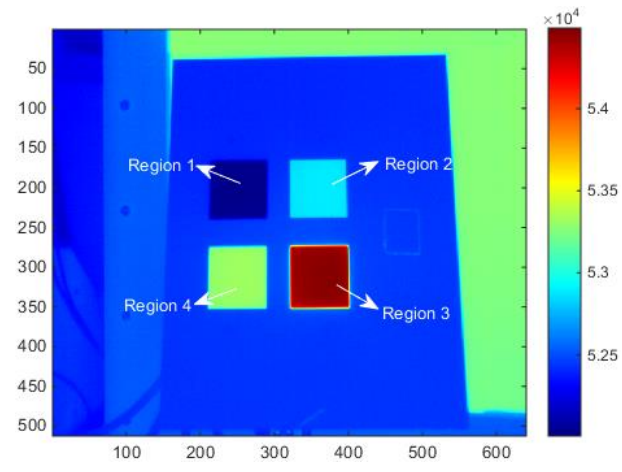

Figure 1. Four element Peltier blackbody

Firstly, four pixels are picked up from respective region 
$(1,2,3,4)$. Then the changes of FPA temperature, housing temperature and four pixels' gray values via time are shown in Figure 2.
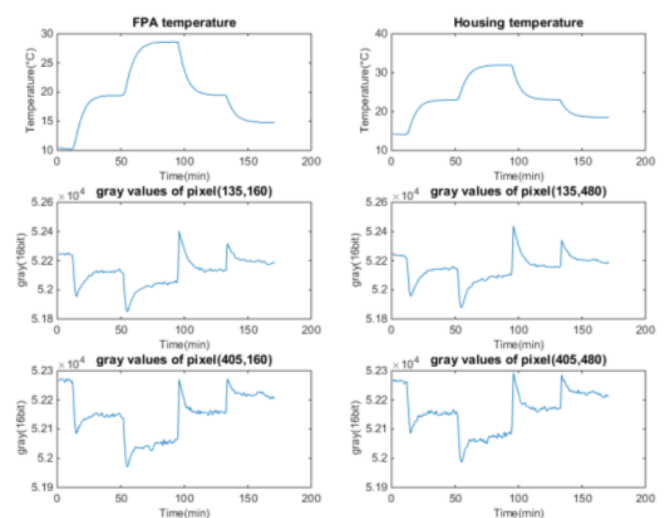

Figure 2. FPA temperature, housing temperature and four pixels' gray values via time

In a next step, every image is classified to one of the following categories, according to absolute changes of FPA temperature over time: stable $\left(0^{\circ} \mathrm{C} / \mathrm{min}\right)$, relatively stable $\left(<0.5^{\circ} \mathrm{C} / \mathrm{min}\right)$, unstable $\left(>0.5^{\circ} \mathrm{C} / \mathrm{min}\right)$. The relation between the FPA temperatures and the gray values from stable images is established by applying a second-order polynomial (shown in Figure 3):
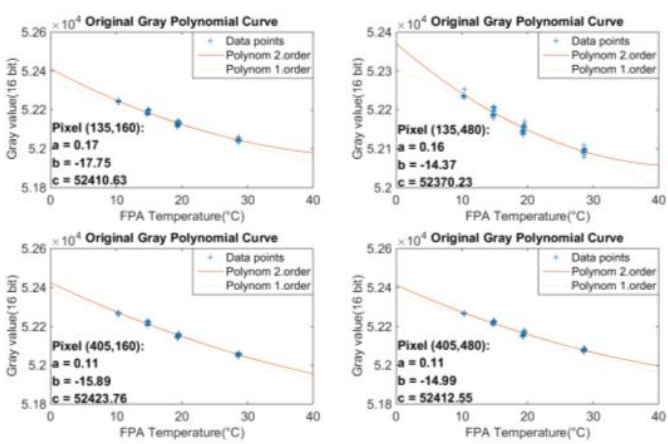

Figure 3. Second-order polynomial parameters determination all of the sequential images are projected into this reference level using Eq. (1):

$$
G_{c}=\mathrm{a}\left(T_{r e f}^{2}-T_{0}^{2}\right)+b\left(T_{\text {ref }}-T_{0}\right)+G_{0}
$$

where $G_{o}=$ original digital output of the camera

$T_{0}=$ current FPA temperature

$T_{\text {ref }}=$ reference FPA temperature

$G_{c}=$ corrected digital response

$\mathrm{a}, \mathrm{b}=$ second order polynomial parameters

After this correction, in terms of the stable and relatively stable images, they are corrected into the same gray level in general. But there are still large deviation for unstable images. However, we found that there is rough third order polynomial relation between temporal difference of FPA temperature and above corrected images. Therefore, the parameters of thirdorder polynomial can be determined as shown in Figure 4.
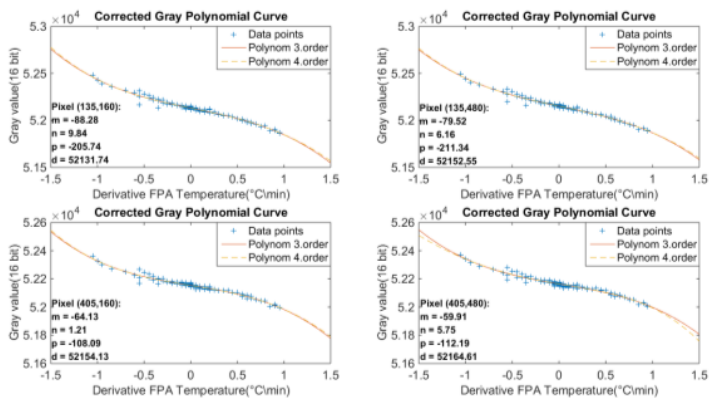

Figure 4. Third-order polynomial parameters determination

All of the above corrected images are modified again using Eq. (2), (3):

We take one of the stable FPA temperature as reference,

$$
\begin{aligned}
& G_{f}=G_{C}-\left(\mathrm{m} \cdot \Delta T_{F P A}{ }^{3}+n \cdot \Delta T_{F P A}{ }^{2}+p \cdot \Delta T_{F P A}\right) \\
& \left\{\begin{array}{c}
\Delta T_{F P A}(i)=T_{F P A}(i+1)-T_{F P A}(i) \\
\Delta T_{F P A}(i)=\left(T_{F P A}(i+2)-T_{F P A}(i)\right) / 2 \quad \text { if } i=1>1 \& \& i<\text { end } \\
\Delta T_{F P A}(i)=T_{F P A}(i)-T_{F P A}(i-1)
\end{array}\right.
\end{aligned}
$$

where $G_{c}=$ result of previous correction

$\mathrm{m}, \mathrm{n}, \mathrm{p}=$ third order polynomial parameters

$\Delta T_{F P A}=$ temporal difference of FPA temperature $i=$ index of image sequence

$$
G_{f}=\text { the final correction result }
$$

After this third order polynomial correction, the range of the image gray fluctuation is much smaller, then all of the digital outputs are considered to be only object 
radiance related.

In addition, all of these polynomial parameters above including second order (three parameters) and third order (four parameters) are pixel-related and timeinvariant, which actually represent matrices whose dimensions match the pixel count of the image. All of the FPA temperature-dependent correction above uses only the digital output and the FPA temperature under the condition of constant blackbody temperature without the requirement of specific blackbody temperature.

Finally, radiometric calibration is implemented to convert image gray values into object temperature values. Radiometric calibration regression coefficients of the camera: R, B, F, O are determined with a Planck curve Eq. (4) using at least four known object temperature under reference FPA temperature.

$$
V_{O}=\frac{\mathrm{R}}{e^{\frac{B}{T_{O}}}-F}+O
$$

Therefore, the inverse function Eq. (5) serves to convert image gray values into object temperature $T_{O}$ :

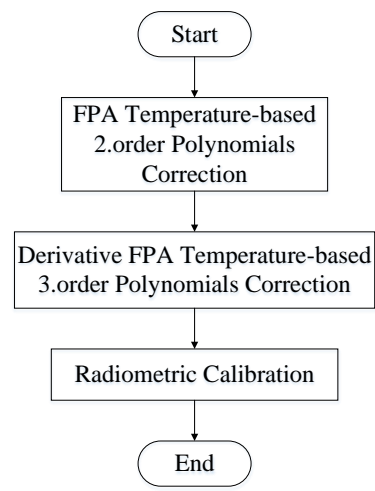

(a) My workflow

$$
T_{O}=\frac{\mathrm{B}}{\ln \left(\frac{R}{V_{O}-O}+F\right)}
$$

\subsection{Application of the Method}

The workflow to use the proposed method is shown in Figure 5(a). Firstly, the sequential images and FPA temperatures are saved at the same time. Next, the FPA temperature-based second-order polynomial model is used to project all of the sequential images into the reference level under the reference FPA temperature. Then, all of the above corrected images are modified again to remove the influence caused by fast changing FPA temperature using derivative PFA temperaturebased third-order polynomial correction. Finally, the normally stabilized response is radiometric calibrated by applying Planck model to get the final object surface temperature.

At the same time, we compare our results with the results provided by FLIR GEV whose workflow is shown in Figure 5(b). Compared with our method, FLIR GEV applies shutter-based temperaturedependent method to remove the response from FPA temperature.

Figure 5. Workflow of two methods

The time-related calibrated result and deviation between the calculated temperature and actual blackbody temperature are presented in Figure 6, which shows that our method is much better than FLIR GEV. Our method has a low absolute mean error $\left(0.23^{\circ} \mathrm{C}\right.$, $0.51^{\circ} \mathrm{C}, 0.08^{\circ} \mathrm{C}, 0.40^{\circ} \mathrm{C}$ ) and a low absolute maximum error $\left(1.45^{\circ} \mathrm{C}, 1.50^{\circ} \mathrm{C}, 0.57^{\circ} \mathrm{C}, 0.77^{\circ} \mathrm{C}\right)$ for all of the regions.. On the other side, the FLIR GEV has a larger absolute mean error $\left(1.17^{\circ} \mathrm{C}, 0.92^{\circ} \mathrm{C}, 0.56^{\circ} \mathrm{C}, 0.74^{\circ} \mathrm{C}\right)$ and also a bigger absolute maximum error $\left(4.81^{\circ} \mathrm{C}\right.$, $\left.2.64^{\circ} \mathrm{C}, 1.49^{\circ} \mathrm{C}, 2.02^{\circ} \mathrm{C}\right)$. Note that the final temperature deviation includes a maximum $\pm 0.15^{\circ} \mathrm{C}$ 
uncertainty for the blackbody.

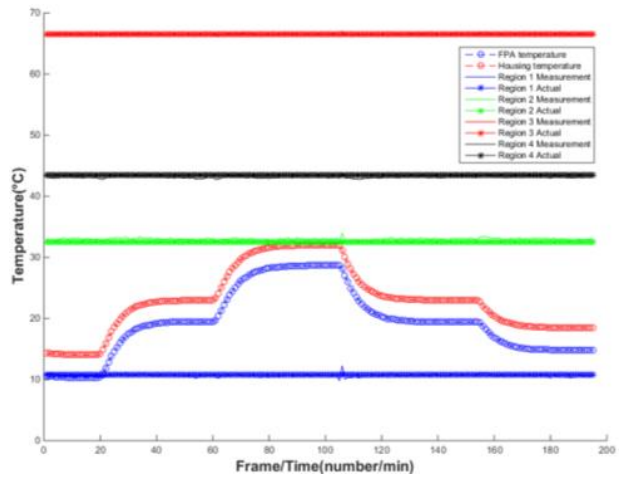

(a) Calibrated result of our method

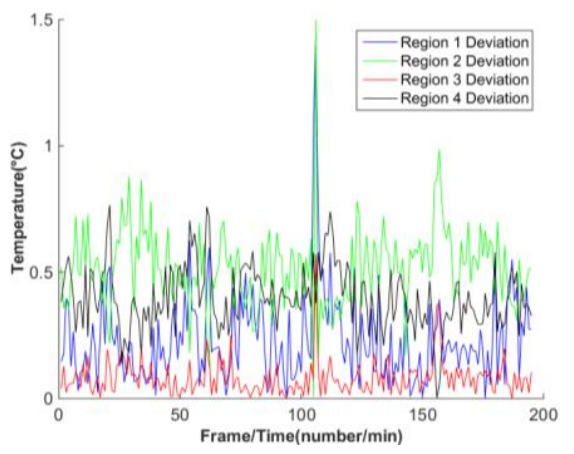

(c) Temperature deviation of our method

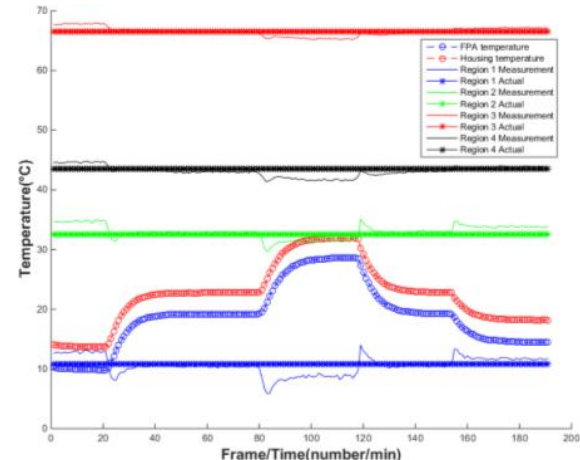

(b) Calibrated result of FLIR GEV

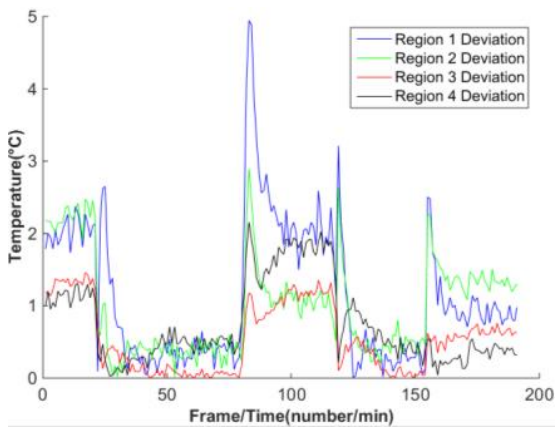

(d) Temperature deviation of FLIR GEV

Figure 6. Four element blackbody experimental results provided by our method and FLIR GEV

Another experiment is implemented to testify my proposed method. During the experiment, compared with four element Peltier blackbody, a larger water bath blackbody with only one constant temperature is used to testify the effectiveness of our method. All of the parameters (including second-order polynomial, thirdorder polynomial and radiometric calibration R, B, F,
O) applied here come from the calculation of four element blackbody experiment. Time-related calibrated result is presented in Figure 7, which proves the effectiveness and stability of our method. Note that the maximum changing speed of FPA temperature is almost the same in these two experiments.

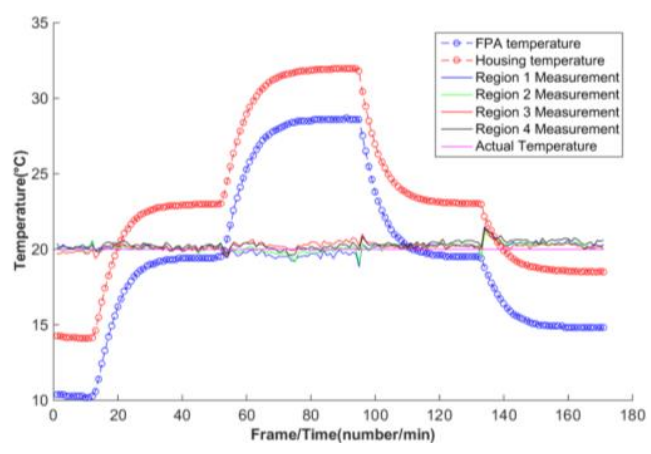

(a) Calibrated result of our method

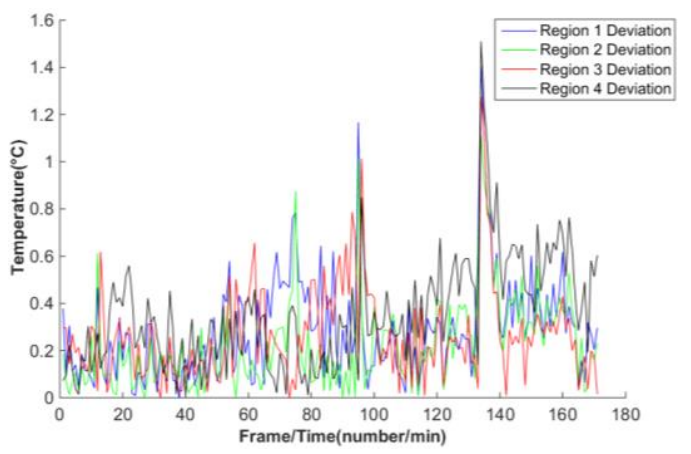

(b) Temperature deviation of our method

Figure 7 . Water bath blackbody experimental results provided by our method

\section{CONCLUSION}

Traditional shutter-based techniques such as software FLIR GEV always assumes that FPA temperature accurately describes the shutter temperature, however, this assumption is not valid any more when FPA temperature is changing fast. In fact, when thermal 
camera is used in the terrestrial application, such as building thermal leakage detection, water contamination monitoring, we care more about temperature difference which represents thermal leakage or sewage source rather than accurate temperature. Therefore, the large deviation range provided by FLIR GEV would probably lead to faulty judgment. On the other side, our method is more efficient for fast changing FPA temperature data.

Furthermore, our method could still be improved in the future by analyzing the relatively large error existed at some of the beginning stages when fast changing FPA temperatures happen. In addition, whether this method is stable or not still needs more cameras to testify.

\section{ACKNOWLEDGEMENTS}

The research work in this paper has been funded by Chinese Scholarship Council and .... In addition, authors would like to acknowledge Dipl. Sandmann, Dr. Budzier and Professor Gerlach (from Solid-State Electronics Laboratory, Dresden) for providing blackbody, chamber and great support.

\section{REFERENCE}

Ambrosia V G, Wegener S S, Sullivan D V, et al. Demonstrating UAV-acquired real-time thermal data over fires $[\mathrm{J}]$. Photogrammetric engineering \& remote sensing, 2003, 69(4): 391-402.

Bhan R K, Saxena R S, Jalwani C R, et al. Uncooled infrared microbolometer arrays and their characterisation techniques[J]. Defence Science Journal, 2009, 59(6): 580.

Budzier H, Gerlach G. Calibration of uncooled thermal infrared cameras $[\mathrm{J}]$. Journal of Sensors and Sensor Systems, 2015, 4(1): 187-197.

Hoegner L, Tuttas S, Stilla U. 3D building reconstruction and construction site monitoring from RGB and TIR image sets[C]//Electronics and Telecommunications (ISETC), 2016 12th IEEE International Symposium on. IEEE, 2016: 305-308.
Johnson J E, Shaw J A, Lawrence R, et al. Long-wave infrared imaging of vegetation for detecting leaking CO2 gas [J]. Journal of Applied Remote Sensing, 2012, 6(1): 063612-063612.

Kruse P W. Uncooled thermal imaging: arrays, systems, and applications [M]. SPIE press, 2001.

Lega M, Napoli R M A. Aerial infrared thermography in the surface waters contamination monitoring [J]. Desalination and water treatment, 2010, 23(1-3): 141151.

Liang K, Yang C, Peng L, et al. Nonuniformity correction based on focal plane array temperature in uncooled long-wave infrared cameras without a shutter [J]. Applied Optics, 2017, 56(4): 884-889.

Liu Z, Blasch E, Xue Z, et al. Objective assessment of multiresolution image fusion algorithms for context enhancement in night vision: a comparative study [J]. IEEE transactions on pattern analysis and machine intelligence, 2012, 34(1): 94-109.

Niklaus F, Vieider C, Jakobsen H. MEMS-based uncooled infrared bolometer arrays: a review[C]//Photonics Asia 2007. International Society for Optics and Photonics, 2007: 68360D-68360D-15.

Nugent P W, Shaw J A, Pust N J. Correcting for focalplane-array temperature dependence in microbolometer infrared cameras lacking thermal stabilization[J]. Optical Engineering, 2013, 52(6): 061304-061304.

Nugent P W, Shaw J A, Pust N J. Radiometric calibration of infrared imagers using an internal shutter as an equivalent external blackbody [J]. Optical Engineering, 2014, 53(12): 123106-123106.

Socolinsky D A, Selinger A, Neuheisel J D. Face recognition with visible and thermal infrared imagery [J]. Computer vision and image understanding, 2003, 91(1): 72-114. 
Tempelhahn A, Budzier H, Krause V, et al. Shutter-less calibration of uncooled infrared cameras [J]. Journal of Sensors and Sensor Systems, 2016, 5(1): 9.

Torres S N, Pezoa J E, Hayat M M. Scene-based nonuniformity correction for focal plane arrays by the method of the inverse covariance form [J]. Applied optics, 2003, 42(29): 5872-5881.

Thurairajah B, Shaw J A. Cloud statistics measured with the infrared cloud imager (ICI) [J]. IEEE Transactions on Geoscience and Remote Sensing, 2005, 43(9): 2000-2007.

Westfeld P, Mader D, Maas H G. Generation of tirattributed $3 \mathrm{~d}$ point clouds from uav-based thermal imagery $[\mathrm{J}]$. Photogrammetrie-FernerkundungGeoinformation, 2015, 2015(5): 381-393. 